\begin{tabular}{|c|c|}
\hline Title & Cross polarized excitons in double-wall carbon nanotubes \\
\hline Author(s) & Tomio, Y uh; Suzuura, Hidekatsu; A ndo, Tsuneya \\
\hline Citation & $\begin{array}{l}\text { Physical Review B, } 86(24), 245428 \\
\text { https://doi.org/10.1103/Phy sRevB.86.245428 }\end{array}$ \\
\hline Issue Date & 2012-12-15 \\
\hline Doc URL & http:/hdl .handle.net/2115/51780 \\
\hline Rights & @2012 A merican Physical Society \\
\hline Type & article \\
\hline File Information & PRB86-24_245428.pdf \\
\hline
\end{tabular}

Instructions for use 


\title{
Cross-polarized excitons in double-wall carbon nanotubes
}

\author{
Yuh Tomio, ${ }^{1, *}$ Hidekatsu Suzuura, ${ }^{1}$ and Tsuneya Ando $^{2}$ \\ ${ }^{1}$ Division of Applied Physics, Graduate School of Engineering, Hokkaido University, Sapporo 060-8628, Japan \\ ${ }^{2}$ Department of Physics, Tokyo Institute of Technology, 2-12-1 Ookayama, Tokyo 152-8551, Japan
}

(Received 12 October 2012; published 26 December 2012)

\begin{abstract}
Optical absorption in double-wall carbon nanotubes for light polarized perpendicular to the tube axis is studied by taking into account exciton effects and depolarization effects within an effective-mass theory. The Coulomb interaction is suppressed by not only intrawall screening effects but also interwall screening, leading to the reduction of exciton binding energies and band gaps. When two tubes are both semiconducting, a clear exciton peak still survives even under depolarization effects for the outer tube, but the exciton peak of the inner tube has an asymmetric Fano line shape due to the coupling with continuum states of the outer tube. When a double-wall nanotube contains a metallic tube, either inner or outer, the exciton of the semiconducting tube loses its peak structure under depolarization effects.
\end{abstract}

DOI: 10.1103/PhysRevB.86.245428

PACS number(s): 78.67.Ch, 73.22.Lp, 71.35.Cc

\section{INTRODUCTION}

A carbon nanotube is a cylindrically rolled-up graphene sheet and has intensively been investigated for its unique physical properties ${ }^{1-3}$ since the discovery. It attracts much attention not only from viewpoints of fundamental physics, but also from those of device applications. The optics of carbon nanotubes is of great interest in that the optical responses are dominated by a small number of exciton states ${ }^{4-11}$ and this essentially reflects the feature of the one-dimensional Wannier exciton with the binding energy, which is unusually larger than that in bulk semiconductor systems. The purpose of this paper is to study cross-polarized excitons excited by the light with polarization perpendicular to the tube axis in double-wall carbon nanotubes by taking interwall screening and depolarization effects into account.

The screening of the Coulomb interaction changes exciton energies unconventionally in nanotubes. For example, antiscreening takes place in semiconducting single-wall carbon nanotubes and enhances the binding energies of excited exciton states. $^{12}$ In photoluminescence studies ${ }^{13,14}$ of double-wall nanotubes, the energies of the optically active exciton states of the inner tubes show a red shift from those of single-wall tubes with the same chiral indices, where the effective dielectric screening is expected to be modified by the presence of the surrounding outer tube, that is, environmental effects. ${ }^{15-18}$ Double-wall tubes are the simplest systems of multiwall carbon nanotubes and are suitable for studying the interactions between different walls. ${ }^{19-26}$ In a previous paper, ${ }^{27}$ we have shown that the excitons in double-wall carbon nanotubes show a red shift caused by the interwall screening.

For single-wall carbon nanotubes, the dynamical conductivity for the light with the polarization perpendicular to the tube axis is considerably reduced by induced depolarization in comparison with that for the light with parallel polarization, while exciton peaks clearly exist in semiconducting nanotubes due to the strong Coulomb interaction. ${ }^{28-36}$ In this study, we systematically examine effects of interwall screening and depolarization on excitons and optical spectra in double-wall tubes based on the effective-mass theory within a static screened Hartree-Fock approximation. We focus on excitons in a semiconducting nanotube contained in a double-wall nanotube where the other coaxial nanotube can be a semiconductor or a metal.

The paper is organized as follows: In Sec. II, we briefly discuss how to calculate dynamical conductivity describing exciton absorption in the cross-polarization geometry in multiwall nanotubes. In Sec. III, we focus on the optical spectra in double-wall tubes and give analysis based on a simple model to clarify depolarization effects and the Fano effect appearing in absorption spectra. In Sec. IV, the diameter dependence of the exciton and the band gap is presented, exhibiting how exciton properties reflect the interwall screening and the depolarization, which are dependent on the interwall distance. The results are discussed and a short summary is given in Sec. V. In Appendix A, the effective dielectric function giving depolarization effects is derived. In Appendix B, the relation between the interwall screening and effects of environmental dielectric material is discussed.

\section{FORMULATION}

In this section, the method to calculate dynamical conductivity in the cross-polarization geometry is given for multiwall nanotubes. It is a straightforward extension of the method in the parallel geometry discussed previously. ${ }^{27}$

The electronic states around the $K$ point in a graphene sheet are described by the $\boldsymbol{k} \cdot \boldsymbol{p}$ equation,, $37-42$

$$
\gamma(\boldsymbol{\sigma} \cdot \hat{\boldsymbol{k}}) \boldsymbol{F}(\boldsymbol{r})=\varepsilon \boldsymbol{F}(\boldsymbol{r}),
$$

where $\gamma$ is a band parameter, related to nearest-neighbor hopping integral $\gamma_{0}$ through $\gamma=(\sqrt{3} / 2) a \gamma_{0}$ with the lattice constant $a=2.46 \AA ; \sigma=\left(\sigma_{x}, \sigma_{y}\right)$ is the Pauli spin matrix; and $\hat{\boldsymbol{k}}=\left(\hat{k}_{x}, \hat{k}_{y}\right)=-i \nabla$ is a wave-vector operator. What specifies the structure of a nanotube is the chiral vector $\boldsymbol{L}$ representing the periodicity in the circumference direction. The energy bands in the carbon nanotubes of diameters much larger than the lattice constant are obtained by simply imposing the boundary condition in the circumference direction $\boldsymbol{F}(\boldsymbol{r}+$ $\boldsymbol{L})=\boldsymbol{F}(\boldsymbol{r}) \exp (-2 \pi i v / 3)$, where $v$ is an integer $(v=0$ or \pm 1$)$ determined by the structure. The nanotube becomes a metal for $v=0$ or a semiconductor for $v= \pm 1$. The energy bands are specified by three indices $(s, n, k)$, where $s$ is equal to + for the 


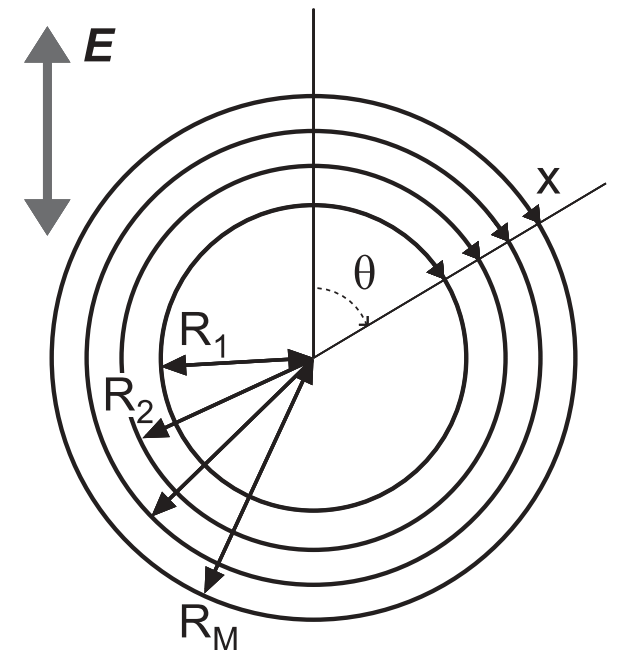

FIG. 1. A schematic illustration of a multiwall carbon nanotube with $M$ walls where $R_{j}$ is the radius of the $j$ th wall, and the polarized electric field perpendicular to the tube axis.

conduction band and to - for the valence band, respectively, $n$ is an integer giving the discrete wave vector along the circumference direction ( $x$ axis) (i.e., angular momentum) and $k$ shows the wave number in the tube axis direction ( $y$ axis).

Consider a multiwall structure containing coaxial singlewall nanotubes of radii $R_{1}, R_{2}, \ldots, R_{M}$ as illustrated in Fig. 1. In general, the lattice structure of each nanotube is incommensurate with that of adjacent walls. ${ }^{43,44}$ This makes interwall electron hopping negligibly small as a result of the cancellation of interwall coupling between each extended state in the absence of disorder. ${ }^{45-48}$ Therefore, we neglect the interwall transfer of electrons in this study, while interwall screening is properly taken into consideration.

Then every electron is considered to be confined in a singlewall nanotube so that the wave function for nanotube with radius $R_{j}$ is written as

$$
\boldsymbol{F}_{s n k}^{R_{j}}(\boldsymbol{r})=\frac{1}{\sqrt{A L_{j}}} \exp \left[i \kappa_{v_{j}}(n) x+i k y\right] \boldsymbol{F}_{s n k}^{v_{j}},
$$

where $A$ is the length of the nanotube, the circumference length $L_{j}=2 \pi R_{j}, v_{j}=0, \pm 1$, and

$$
\begin{gathered}
\boldsymbol{F}_{s n k}^{v_{j}}=\frac{1}{\sqrt{2}}\left[\begin{array}{c}
b_{v_{j}}(n, k) \\
s
\end{array}\right], \\
\kappa_{v_{j}}(n)=\frac{2 \pi}{L_{j}}\left(n-\frac{v_{j}}{3}\right), \\
b_{v_{j}}(n, k)=\frac{\kappa_{v_{j}}(n)-i k}{\sqrt{\kappa_{v_{j}}(n)^{2}+k^{2}}} .
\end{gathered}
$$

The eigenenergy is given by

$$
\varepsilon_{j, s, n}^{K}(k)=s \gamma \sqrt{\kappa_{\nu_{j}}(n)^{2}+k^{2}} .
$$

For the $K^{\prime}$ point, we have only to replace $k$ with $-k$ and $v$ with $-v$ in Eqs. (3)-(5). This correspondence is as a consequence of the fact that the $K$ and $K^{\prime}$ points are related to each other via the time-reversal operation. ${ }^{49,50}$
The Fourier transform of the Coulomb interaction, with angular momentum $m$ along the circumference and wave number $q$ in the axis direction is given by ${ }^{51-56}$

$$
V_{i j}^{0}(m, q)=2 e^{2} I_{|m|}\left(|q| R_{\min }\right) K_{|m|}\left(|q| R_{\max }\right),
$$

with $R_{\min }=\min \left(R_{i}, R_{j}\right)$ and $R_{\max }=\max \left(R_{i}, R_{j}\right)$, where $I_{m}(t)$ and $K_{m}(t)$ are the modified Bessel function of the first and second kind, respectively. The polarization function of tube $j$ can be written as

$$
P_{j}(m, q, \omega)=\Pi_{j}(m, q, \omega)+\Pi_{j}^{\prime}(m, q, \omega),
$$

where the contributions due to electrons in the vicinity of the $K$ and $K^{\prime}$ points, $\Pi_{j}(m, q, \omega)$, are separated out from those due to other electrons, $\Pi_{j}^{\prime}(m, q, \omega)$. We replace the latter with phenomenological dielectric constant $\kappa$ in such a way that ${ }^{18}$ $V_{j j}^{0}(m, q) \Pi_{j}^{\prime}(m, q, \omega) \simeq \kappa-1$. Then, the effective dielectric function of tube $j$ without interwall screening becomes

$$
\epsilon_{j}(m, q, \omega)=\kappa+V_{j j}^{0}(m, q) \Pi_{j}(m, q, \omega) .
$$

With the inclusion of both inter and intrawall screening, the screened Coulomb interaction in multiwall tubes becomes

$$
V_{i j}(m, q, \omega)=\sum_{\ell}\left[\hat{\epsilon}(m, q, \omega)^{-1}\right]_{i \ell} V_{\ell j}^{0}(m, q),
$$

with

$$
\hat{\epsilon}=\left(\begin{array}{cccc}
\epsilon_{1} & \beta_{12}\left(\epsilon_{2}-1\right) & \ldots & \beta_{1 M}\left(\epsilon_{M}-1\right) \\
\beta_{21}\left(\epsilon_{1}-1\right) & \epsilon_{2} & \ldots & \beta_{2 M}\left(\epsilon_{M}-1\right) \\
\vdots & \vdots & \ddots & \vdots \\
\beta_{M 1}\left(\epsilon_{1}-1\right) & \beta_{M 2}\left(\epsilon_{2}-1\right) & \ldots & \epsilon_{M}
\end{array}\right) \text {, }
$$

where

$$
\beta_{i j}(m, q)=\frac{V_{i j}^{0}(m, q)}{V_{j j}^{0}(m, q)},
$$

as long as the interwall charge transfer can be neglected. This expression for the Coulomb interaction is equivalent to that in the previous study on the light absorption for the polarization parallel to the tube axis. ${ }^{27}$

In this study, we do not consider the exciton fine structures due to intervalley mixing since the Coulomb matrix elements for intervalley scattering, which need large momentum transfer, are much smaller than those for the intravalley scattering. ${ }^{57-60}$ We only consider the exciton formed by an electron-hole pair belonging to the same valley and having the angular momentum in the circumference direction via absorption of a photon with the perpendicular polarization.

For the tube with radius $R_{j}$, the exciton state near the $K$ valley with angular momentum $l$ around the axis is expressed by

$$
|u, l ; j\rangle=\sum_{n, k} \psi_{j, n}^{u, l}(k) c_{j,+, n+l, k}^{K \dagger} c_{j,-, n, k}^{K}|g\rangle,
$$

where $|g\rangle$ is the ground-state wave function and $c_{j, s, n, k}^{K}$ and $c_{j, s, n, k}^{K \dagger}$ are the annihilation and creation operators, respectively, for states specified by $(s, n, k)$ in the $K$ valley. 
Within the static screening approximation, the exciton wave function $\psi_{j, n}^{u, l}(k)$ satisfies the Bethe-Salpeter equation,

$$
\begin{aligned}
E_{j}^{u} \psi_{j, n}^{u, l}(k)= & {\left[\tilde{\varepsilon}_{j,+, n+l}^{K}(k)-\tilde{\varepsilon}_{j,-, n}^{K}(k)\right] \psi_{j, n}^{u, l}(k) } \\
& -\sum_{m, q} V_{(+, n+l, k ;+, m+l, k+q)(-, m, k+q ;-, n, k)}^{j j} \\
& \times \psi_{j, m}^{u, l}(k+q),
\end{aligned}
$$

with

$$
\begin{aligned}
& V_{\left(s_{1}, n+l, k ; s_{2}, m+l, k+q\right)\left(s_{3}, m, k+q ; s_{4}, n, k\right)}^{j j} \\
& =\frac{1}{A} V_{j j}(n-m, q, 0)\left(\boldsymbol{F}_{s_{1}, n+l, k}^{v_{j} \dagger} \cdot \boldsymbol{F}_{s_{2}, m+l, k+q}^{v_{j}}\right) \\
& \quad \times\left(\boldsymbol{F}_{s_{3}, m, k+q}^{v_{j} \dagger} \cdot \boldsymbol{F}_{s_{4}, n, k}^{v_{j}}\right),
\end{aligned}
$$

and the renormalized band dispersion,

$$
\tilde{\varepsilon}_{j, s, n}^{K}(k)=\varepsilon_{j, s, n}^{K}(k)+\Sigma_{j, s, n}^{K}(k),
$$

where $\Sigma_{j, s, n}^{K}(k)$ is the self-energy given by

$$
\begin{aligned}
\Sigma_{j, s, n}^{K}(k)= & -\sum_{m} \sum_{q} V_{(s, n, k ;-, m, k+q)(-, m, k+q ; s, n, k)}^{j j} \\
& \times g_{0}\left[\varepsilon_{j, s, m}^{K}(k+q)\right],
\end{aligned}
$$

in the screened Hartree-Fock approximation.

The polarization function in Eq. (9) is calculated as

$$
\Pi_{j}(m, q, 0)=\Pi_{j}^{K}(m, q)+\Pi_{j}^{K^{\prime}}(m, q),
$$

with

$$
\begin{aligned}
\Pi_{j}^{K}(m, q)= & \frac{2 g_{s}}{A} \sum_{n} \sum_{k}\left|\boldsymbol{F}_{-, n, k}^{v_{j} \dagger} \cdot \boldsymbol{F}_{+, m+n, k+q}^{v_{j}}\right|^{2} \\
& \times \frac{g_{0}\left[\varepsilon_{j,+, m+n}^{K}(k+q)\right] g_{0}\left[\varepsilon_{j,-, n}^{K}(k)\right]}{\varepsilon_{j,+, m+n}^{K}(k+q)-\varepsilon_{j,-, n}^{K}(k)},
\end{aligned}
$$

where $g_{s}=2$ is the spin degeneracy.

In the above formulations, we have introduced the cutoff function

$$
g_{0}(\varepsilon)=\frac{\varepsilon_{c}^{\alpha_{c}}}{|\varepsilon|^{\alpha_{c}}+\varepsilon_{c}^{\alpha_{c}}} .
$$

It contains two parameters $\alpha_{c}$ and $\varepsilon_{c}$, which should be chosen in such a way that we include only the contributions from states in the vicinity of the Fermi level. The appropriate value of $\varepsilon_{c}$ is about the half of the $\pi$ band width $\sim 3 \gamma_{0}$ which gives $\varepsilon_{c} /(2 \pi \gamma / L) \approx 10$ for typical nanotubes with diameter $2 R \sim 1.4 \mathrm{~nm}$. Calculations for slightly different values of these parameters essentially give the same results.

For polarization perpendicular to the tube axis, the dynamical conductivity of tube $j$ is calculated using the Kubo formula $^{61}$ as

$$
\sigma_{j}^{l}(\omega)=\frac{g_{s} \hbar e^{2}}{A L_{j}} \sum_{K K^{\prime}} \sum_{u} \frac{-2 i \hbar \omega\left|\left\langle u, l ; j\left|v_{x}^{l}\right| g\right\rangle\right|^{2}}{E_{j}^{u}\left(E_{j}^{u 2}-\hbar^{2} \omega^{2}-2 i \hbar \omega \Gamma\right)},
$$

where the velocity operator in the circumference direction is given by $v_{x}^{l}=(\gamma / \hbar) \sigma_{x} e^{i l \theta}$ independent of the $K$ and $K^{\prime}$ points, and $\Gamma$ is a phenomenological broadening parameter.

In the parallel-polarization geometry, the interwall coupling of electric fields is quite negligible as long as the wavelength of incident light is much larger than the tube radius for infinite length tubes. This is also very small for finite length multiwall tubes. ${ }^{26}$ In the cross-polarization geometry, on the other hand, the depolarization effect is quite essential and gives rise to the strong interwall coupling of the electric fields for multiwall tubes.

The depolarization effect for cross-polarized exciton states can be obtained by considering self-consistent electric fields. ${ }^{31,62,63}$ Suppose an external electric field is applied in the direction perpendicular to the tube axis. Let $j_{i x}^{l}, E_{i x}^{l}$, and $D_{i x}^{l}$ be the components of the circumference direction of the induced current, effective electric field and external field on a cylinder surface with radius $R_{i}$, respectively. Then, we have

$$
\boldsymbol{E}_{x}^{l}=\hat{\epsilon}^{l}(\omega)^{-1} \boldsymbol{D}_{x}^{l},
$$

where $\quad{ }^{t} \boldsymbol{E}_{x}^{l}=\left(E_{1 x}^{l}, E_{2 x}^{l}, \ldots, E_{M x}^{l}\right) \quad$ and $\quad{ }^{t} \boldsymbol{D}_{x}^{l}=$ $\left(D_{1 x}^{l}, D_{2 x}^{l}, \ldots, D_{M x}^{l}\right)$. The dielectric matrix $\hat{\epsilon}^{l}(\omega)$ for depolarization effects is defined by

$$
\hat{\epsilon}^{l}(\omega)=\hat{R}^{-1} \hat{\epsilon}(l, 0, \omega) \hat{R},
$$

where $\hat{R}$ is a diagonal matrix with $[\hat{R}]_{i j}=R_{i} \delta_{i j}$. The matrix $\hat{\epsilon}(l, 0, \omega)$ has the same form of Eq. (11), while the dielectric function of Eq. (9) due to particle-hole pair productions causing interwall screening should be replaced by that due to the current-induced depolarization field

$$
\epsilon_{j}^{l}(\omega) \equiv \epsilon_{j}(l, 0, \omega)=\kappa+\frac{4 \pi^{2} i|l|}{L_{j} \omega} \sigma_{j}^{l}(\omega),
$$

and $\beta_{i j}(l, 0)=\left(R_{\min } / R_{\max }\right)^{|l|}$. We note that Eq. (22) is equivalent to the equation for static fields in Ref. 64 and we have extended it to the dynamic regime, here. A naive derivation of Eq. (22) is given in Appendix A.

The external field is usually common on all tubes (i.e., $\left.D_{i x}^{l}=D_{x}^{l}\right)$. By using the relation between the current and the electric field $j_{i x}^{l}=\sigma_{i}^{l}(\omega) E_{i x}^{l}$, we can define an effective conductivity as a response to the external field,

$$
j_{i x}^{l}=\tilde{\sigma}_{i}^{l}(\omega) D_{x}^{l},
$$

where

$$
\tilde{\sigma}_{i}^{l}(\omega)=\frac{\sigma_{i}^{l}(\omega)}{\tilde{\epsilon}_{i}^{l}(\omega)},
$$

with the effective dielectric function defined by

$$
\tilde{\epsilon}_{i}^{l}(\omega)^{-1}=\sum_{j}\left[\hat{\epsilon}^{l}(\omega)^{-1}\right]_{i j} .
$$

For perpendicular polarization, the external field in the $x y$ plane is given by $\boldsymbol{D}=(D \sin \theta, 0)$. Then, the Fourier components of the external field and the induced current are written as $D_{x}^{l}=\delta_{l, 1} D /(2 i)-\delta_{l,-1} D /(2 i)$ and $j_{i x}^{l}=$ $\delta_{l, 1} \tilde{\sigma}_{i}^{l=1}(\omega) D /(2 i)-\delta_{l,-1} \tilde{\sigma}_{i}^{l=-1}(\omega) D /(2 i)$, respectively. The total absorption of a multiwall tube is given by the sum of that of each tube,

$$
\begin{aligned}
P(\omega) & =\frac{1}{2} \sum_{i} \sum_{l} \int_{0}^{2 \pi R_{i}} d x \operatorname{Re}\left[j_{i x}^{l} E_{i x}^{l *}\right] \\
& =\frac{1}{4} 2 \pi \bar{R} \operatorname{Re}[\tilde{\sigma}(\omega)] D^{2},
\end{aligned}
$$


where the effective dynamical conductivity $\tilde{\sigma}(\omega)$ is defined by

$$
\begin{gathered}
\tilde{\sigma}(\omega)=\bar{R}^{-1} \sum_{i} R_{i} \tilde{\sigma}_{i}(\omega), \quad \bar{R}=\frac{1}{M} \sum_{j=1}^{M} R_{j}, \\
\tilde{\sigma}_{i}(\omega)=\frac{1}{2}\left[\frac{\sigma_{i}^{l=1}(\omega)}{\left|\tilde{\epsilon}_{i}^{l=1}(\omega)\right|^{2}}+\frac{\sigma_{i}^{l=-1}(\omega)}{\left|\tilde{\epsilon}_{i}^{l=-1}(\omega)\right|^{2}}\right] .
\end{gathered}
$$

The frequency $\omega_{0}$ of the exciton resonance with depolarization effects taken into consideration is given by a zero of $\tilde{\epsilon}_{i}^{l}(\omega)$, that is,

$$
\operatorname{det}\left[\hat{\epsilon}^{l}\left(\omega_{0}\right)\right]=0 .
$$

Without the depolarization effect, the absorption is given by replacing $\tilde{\sigma}_{i}(\omega)$ with $\sigma_{i}(\omega)$ defined by

$$
\sigma_{i}(\omega)=\frac{1}{2}\left[\sigma_{i}^{l=1}(\omega)+\sigma_{i}^{l=-1}(\omega)\right] .
$$

The conductivity $\tilde{\sigma}_{i}(\omega)$ above gives the current induced by the external electric field $D$, which is the field infinitely far away from the nanotube. Actually, the field in the vicinity of the multiwall tube is reduced from $D$ because of the presence of $\kappa$ associated with screening due to electrons except those in the vicinity of the $K$ and $K^{\prime}$ points in each wall. The field just on the surface of the outermost tube is given by $E=D / \tilde{\kappa}$, with the use of another effective dielectric constant

$$
\tilde{\kappa}=\tilde{\epsilon}_{M}^{l= \pm 1},
$$

where we should omit the contribution proportional to $\sigma_{j}^{l}(\omega)$ in Eq. (24). In the following sections, we shall use the rescaled conductivities so as to represent the absorption with respect to field $E$ instead of $D$,

$$
\begin{aligned}
& \tilde{\sigma}_{i}^{\prime}(\omega)=\tilde{\kappa}^{2} \tilde{\sigma}_{i}(\omega), \\
& \tilde{\sigma}^{\prime}(\omega)=\tilde{\kappa}^{2} \tilde{\sigma}(\omega) .
\end{aligned}
$$

\section{DOUBLE-WALL TUBES}

Hereafter, we consider a double-wall tube with radii $R$ and $R^{\prime}$. The static screened Coulomb interaction between two electrons on the cylinder surface with radius $R$ is explicitly calculated as

$$
V_{R R}(m, q)=\frac{V_{R R}^{0}(m, q)}{\tilde{\epsilon}_{R}(m, q)},
$$

with the effective dielectric function including interwall screening

$$
\begin{aligned}
\tilde{\epsilon}_{R}(m, q)= & \epsilon_{R}(m, q, 0) \\
& +\frac{\tau_{m}(q)\left[\epsilon_{R^{\prime}}(m, q, 0)-1\right]}{\epsilon_{R^{\prime}}(m, q, 0)-\tau_{m}(q)\left[\epsilon_{R^{\prime}}(m, q, 0)-1\right]},
\end{aligned}
$$

where

$$
\begin{aligned}
\tau_{m}(q) & =\beta_{R R^{\prime}}(m, q) \beta_{R^{\prime} R}(m, q) \\
& =\frac{I_{|m|}\left(|q| R_{\min }\right) K_{|m|}\left(|q| R_{\max }\right)}{K_{|m|}\left(|q| R_{\min }\right) I_{|m|}\left(|q| R_{\max }\right)} .
\end{aligned}
$$

The effective dielectric function $\tilde{\varepsilon}_{R^{\prime}}(m, q)$ and the screened potential $V_{R^{\prime} R^{\prime}}(m, q)$ for the other tube with $R^{\prime}$ can be obtained by exchanging $R$ and $R^{\prime}$.
The interwall screening comes from the second term of Eq. (37) via the presence of the interwall Coulomb interaction. We can easily show $0 \leqslant \tau_{m}(q) \leqslant 1$. In the long-wavelength case $q R \ll 1$ and $q R^{\prime} \ll 1$, we have $\tau_{m}(q) \simeq\left(R_{\min } / R_{\max }\right)^{2|m|}$ for $m \neq 0$. For $m=0, \tau_{m}(q)$ has a logarithmic singularity and its derivative diverges at $q=0$ except in the special case of $R=R^{\prime} .^{27}$ In the short-wavelength case $q R \gg 1$ and $q R^{\prime} \gg 1$, we have $\tau_{m}(q) \simeq \exp \left(-2 q\left|R-R^{\prime}\right|\right)$. This implies that the interwall screening effect becomes weaker for the short-range scale less than $\left|R-R^{\prime}\right|$. The interaction reduces to that in single-wall tubes, $V_{R R}(m, q)=V_{R R}^{0}(m, q) / \epsilon_{R}(m, q, 0)$, for $R_{\max } / R_{\min }=\infty$.

The effective dielectric function (27) with $l= \pm 1$ can be explicitly written as

$$
\begin{gathered}
\tilde{\epsilon}_{R}^{l}(\omega)=\epsilon_{R}^{l}(\omega) \epsilon_{R^{\prime}}^{l}(\omega)-\tau\left[\epsilon_{R}^{l}(\omega)-1\right]\left[\epsilon_{R^{\prime}}^{l}(\omega)-1\right], \\
\tilde{\epsilon}_{R^{\prime}}^{l}(\omega)=\epsilon_{R^{\prime}}^{l}(\omega)+\frac{\tau\left[\epsilon_{R}^{l}(\omega)-1\right]}{\epsilon_{R}^{l}(\omega)-\tau\left[\epsilon_{R}^{l}(\omega)-1\right]},
\end{gathered}
$$

for $R \leqslant R^{\prime}$ where

$$
\tau \equiv \tau_{l}(0)=\left(\frac{R}{R^{\prime}}\right)^{2|l|} .
$$

The exciton resonance $\omega_{0}$ is given by a zero of $\tilde{\epsilon}_{i}^{l}(\omega)$,

$$
\epsilon_{R}^{l}\left(\omega_{0}\right) \epsilon_{R^{\prime}}^{l}\left(\omega_{0}\right)-\tau\left[\epsilon_{R}^{l}\left(\omega_{0}\right)-1\right]\left[\epsilon_{R^{\prime}}^{l}\left(\omega_{0}\right)-1\right]=0 .
$$

The effective dielectric constant on the surface of the outer tube is written as

$$
\tilde{\kappa}=\frac{\kappa^{2}-\tau(\kappa-1)^{2}}{\kappa-\tau(\kappa-1)} .
$$

We have $\tilde{\epsilon}_{R}^{l}(\omega)=\tilde{\epsilon}_{R^{\prime}}^{l}(\omega)=2 \epsilon_{R}^{l}(\omega)-1$ and $\tilde{\kappa}=2 \kappa-1$ for $R=R^{\prime}$ and $\tilde{\epsilon}_{R}^{l}(\omega)=\epsilon_{R}^{l}(\omega) \epsilon_{R^{\prime}}^{l}(\omega), \tilde{\epsilon}_{R^{\prime}}^{l}(\omega)=\epsilon_{R^{\prime}}^{l}(\omega)$, and $\tilde{\kappa}=\kappa$ for $R \ll R^{\prime}$, as is expected. Further, the dielectric functions (39) and (40) are closely related to that in the presence of environmental dielectric material surrounding or inserted into a single-wall nanotube, respectively. ${ }^{18,65,66}$ The correspondence is discussed in Appendix B.

The strength of the Coulomb interaction is specified by a dimensionless parameter $\left(e^{2} / \kappa L\right) /(2 \pi \gamma / L)$ with $L=2 \pi R$, which is estimated as $\left(e^{2} / \kappa L\right) /(2 \pi \gamma / L) \simeq 0.4 / \kappa$ for $\gamma_{0}=$ $2.7 \mathrm{eV}$. In bulk graphite, we have $\kappa \simeq 2.5$ or $\left(e^{2} / \kappa L\right) /(2 \pi \gamma / L)=0.16$, which is used in the present numerical calculations.

The precise value of $\kappa$ in carbon nanotubes has not been known yet, and previous calculations for parallel polarization show that the exciton energy is almost independent of $\left(e^{2} / \kappa L\right) /(2 \pi \gamma / L)$ for a wide range. 5,18 However, the exciton energy in cross-polarization geometry restricts the interaction parameter, suggesting $0.1<\left(e^{2} / \kappa L\right) /(2 \pi \gamma / L)<$ $0.2 .^{31}$ Furthermore, the energy of the two-photon excited state is more sensitive, putting much narrower bound, $0.15<$ $\left(e^{2} / \kappa L\right) /(2 \pi \gamma / L)<0.16 .^{67}$

Figure 2 shows absorption spectra of a double-wall nanotube consisting of two semiconducting tubes for $R^{\prime} / R=1.4$ with $\varepsilon_{c}(2 \pi \gamma / L)^{-1}=10$, corresponding to typical double-wall tubes with inner diameter $\sim 1 \mathrm{~nm}$. The top panel shows the spectra of two independent single-wall nanotubes. The middle and bottom panels show the spectra without and 


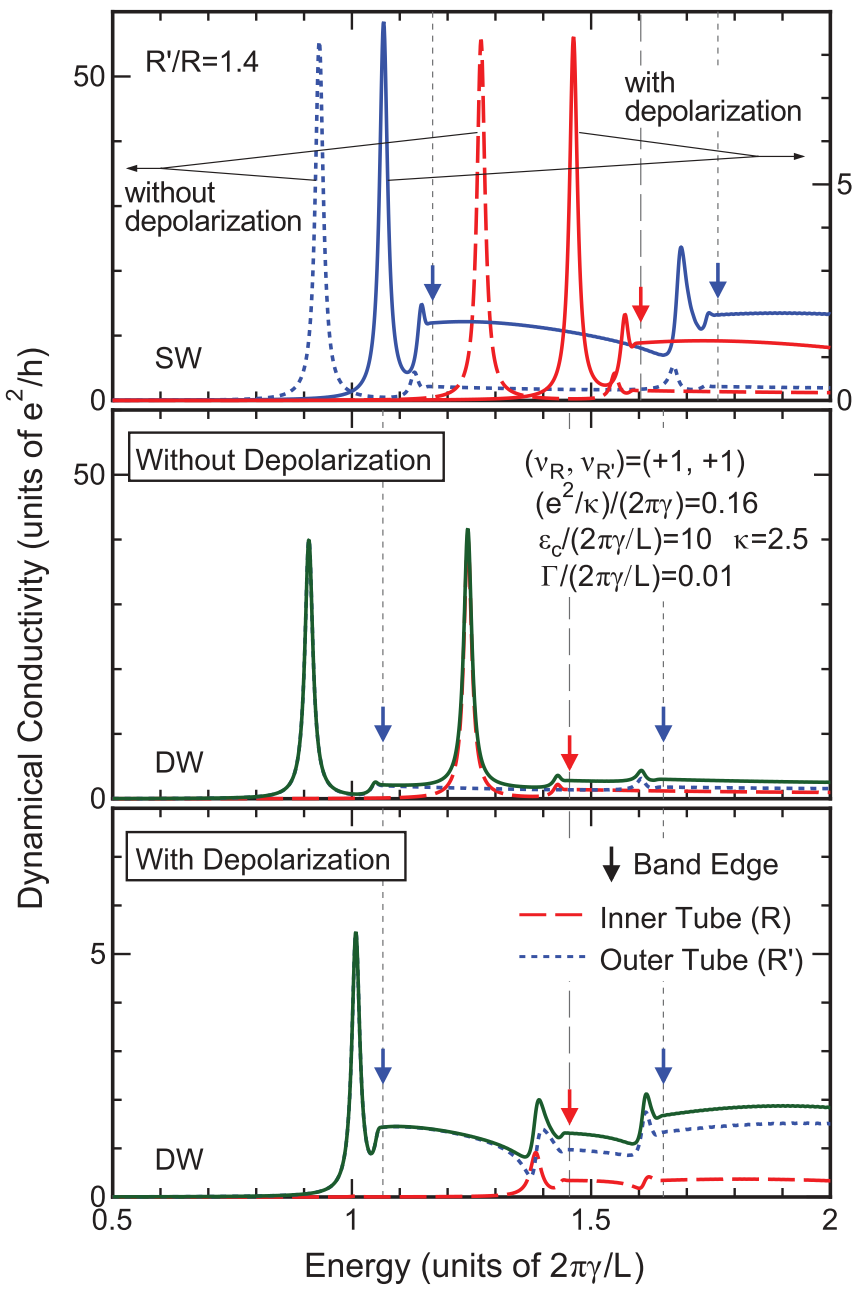

FIG. 2. (Color online) Calculated absorption spectra of a doublewall nanotube consisting of two semiconducting tubes for $R^{\prime} / R=$ 1.4. The top panel shows the spectra with and without depolarization effect for corresponding single-wall tubes. The middle and bottom panels show the spectra in the double-wall tube without and with depolarization effects, respectively. The dashed and dotted curves denote the contribution from the inner and outer tubes, respectively. The arrows denote the bottom of the interband continuum. Phenomenological broadening $\Gamma(2 \pi \gamma / L)^{-1}=0.01$ is introduced.

with depolarization effects, respectively. The vertical arrows indicate the bottom of the interband continuum for each tube. The dashed curves denote the contribution of the inner tube and the dotted lines that of the outer tube.

For the outer nanotube, the band gap decreases considerably due to interwall screening, but the exciton without depolarization is only slightly shifted to the lower energy side. This is due to the cancellation of interwall screening on the band gap and the exciton binding energy as has been discussed previously for parallel geometry. ${ }^{27}$ With the inclusion of depolarization, the exciton peak is shifted to the higher energy side close to the band gap and its intensity is reduced. The qualitative feature of the depolarization effect is essentially the same as in the single-wall tube.

For the inner tube, on the other hand, the exciton peak is strongly modified, although the feature of the band gap and the exciton without depolarization is again the same

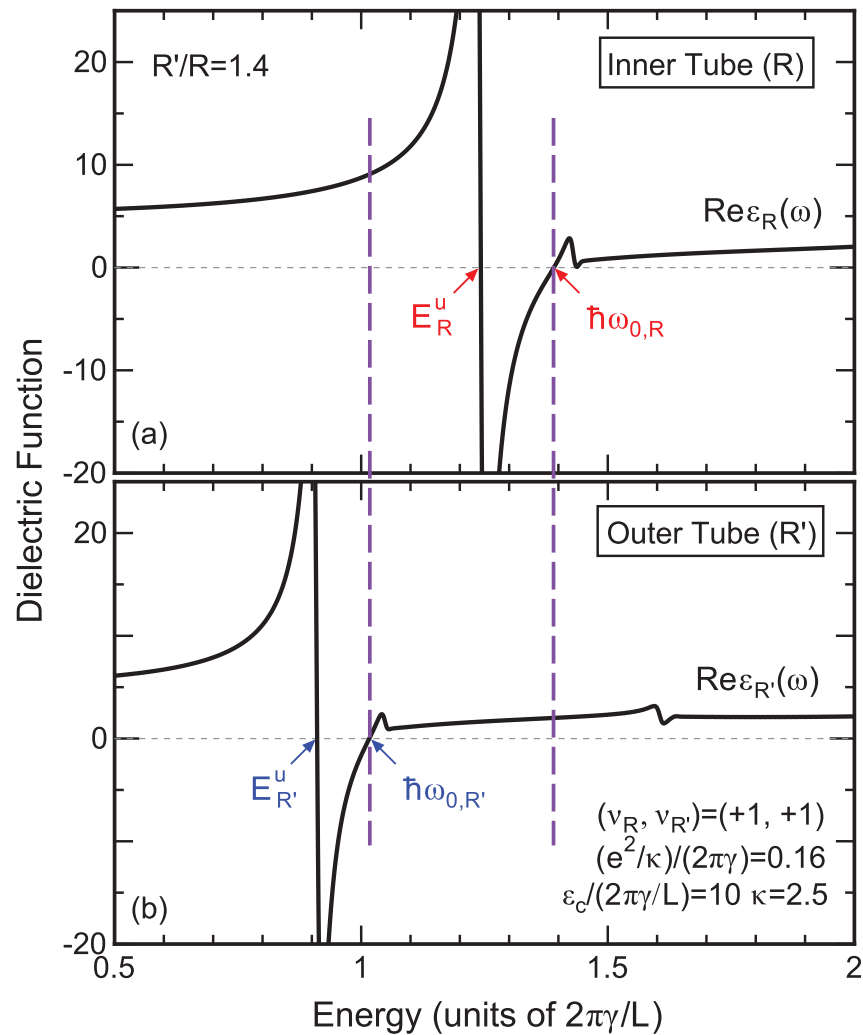

FIG. 3. (Color online) Real part of the dielectric function of (a) semiconducting inner tube $\epsilon_{R}^{l}(\omega)$ and (b) semiconducting outer tube $\epsilon_{R^{\prime}}^{l}(\omega)$ for $R^{\prime} / R=1.4$ (other parameters are the same as in Fig. 2), where $\hbar \omega_{0, i}$ denotes the zero of $\epsilon_{i}^{l}(\omega)$.

as in the parallel geometry. The contribution of the inner tube exhibits a peak structure with symmetric line shape around $\hbar \omega(2 \pi \gamma / L)^{-1}=1.38$ and its peak intensity is much smaller than that of the lowest exciton in the outer tube. The contribution of the outer tube around there shows a strongly asymmetric Fano-like peak, leading to an asymmetric Fano-like structure in the total absorption.

The real part of the dielectric function for each tube is shown for $R^{\prime} / R=1.4$ in Fig. 3. The figure clearly shows that the positions of the excitons without depolarization, $E_{R}^{u}$ and $E_{R^{\prime}}^{u}$, are well separated from each other. This means that we can regard the other tube as a material with effective dielectric constant independent of frequency in order to discuss effects of interwall screening on the depolarization shift of exciton peaks. In this case, we have the effective dielectric constant originating from the inner tube, $\kappa_{\text {in }} \simeq 3 \kappa$, around the exciton peak of the outer tube, leading to reduction of the depolarization effect. For the exciton of the inner nanotube, we have $\kappa_{\text {out }} \simeq \kappa$, showing that $\operatorname{Im} \sigma_{R^{\prime}}^{l}(\omega) \approx 0$ inside the interband continuum.

Figure 4 shows absorption spectra when the outer tube is metallic. There is no exciton peak of the metallic outer tube. This is quite in contrast to the parallel geometry in which a clear exciton feature appears in absorption although the binding energy is much smaller than in semiconducting tubes. ${ }^{68,69}$ The absence of exciton in the present cross-polarization geometry can be ascribed to the vanishing form factor in the Coulomb interaction concerning the electron or hole scattering between metallic linear bands with $n=0,{ }^{68}$ which is closely related to 


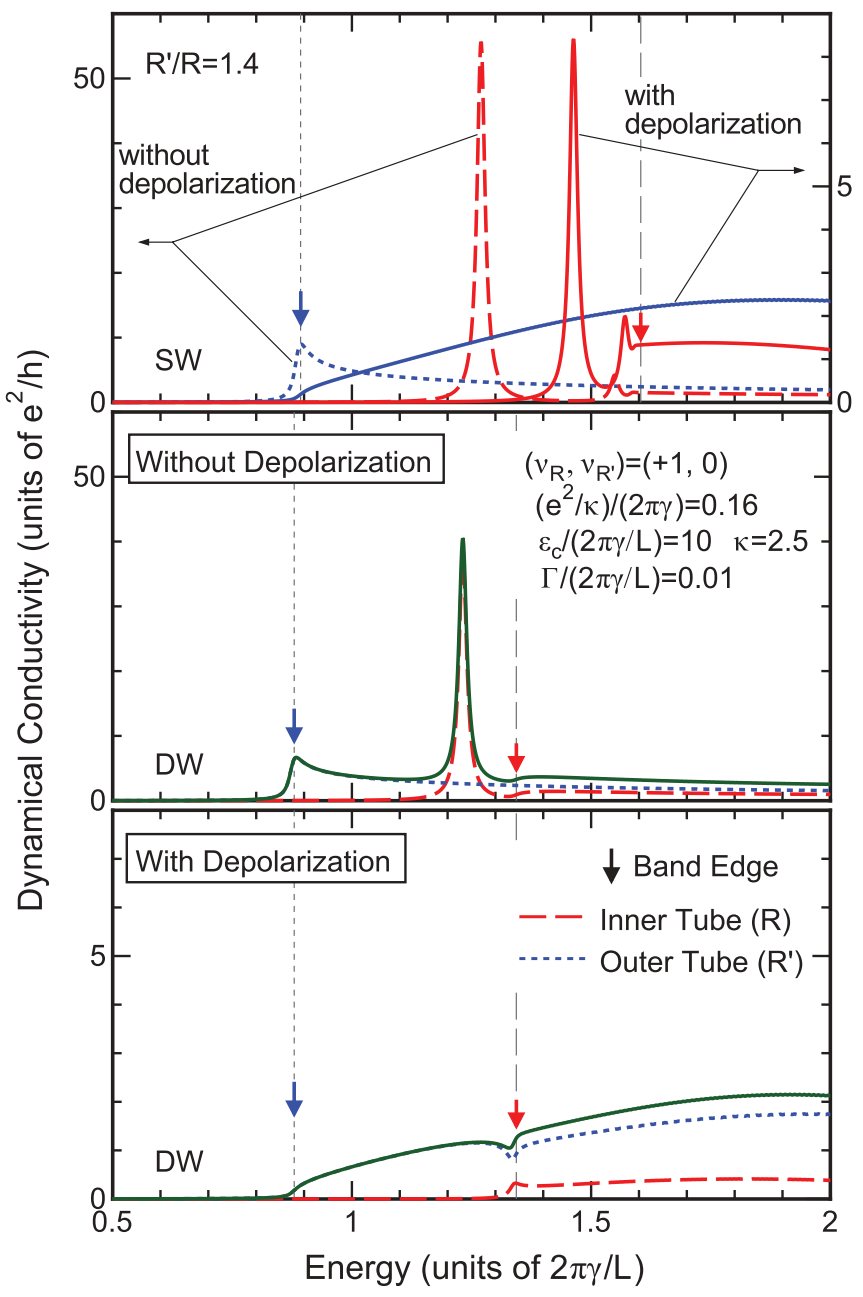

FIG. 4. (Color online) Calculated absorption spectra of a doublewall carbon nanotube consisting of inner semiconducting tube with $R$ and outer metallic tube with $R^{\prime} . R^{\prime} / R=1.4$.

the absence of electron backward scattering in metallic carbon nanotubes. ${ }^{70-72}$ The shift of the band gap in the metallic tube due to interwall screening is quite small. This is the same as in the parallel geometry discussed previously ${ }^{27}$ and presumably due to the fact that the screening is dominated by metallic electrons. The exciton peak of the inner semiconducting nanotube is almost destroyed and remains only as a dip in the total absorption, corresponding to a Fano line shape.

As mentioned above, the exciton associated with the inner nanotube is strongly affected by the presence of the interband continuum in the outer tube and the effect is stronger when the outer tube is metallic. In order to explicitly see this effect, we consider a simple model consisting of a single level and a continuum independent of energy,

$$
\begin{gathered}
\sigma_{R}^{l}(\omega)=\frac{\kappa R \omega}{2 \pi i} \frac{\left(\hbar \omega_{R, p}\right)^{2}}{E_{R}^{u 2}-(\hbar \omega)^{2}-2 i \hbar \omega \Gamma}, \\
\sigma_{R^{\prime}}^{l}(\omega)=\sigma_{c},
\end{gathered}
$$

with

$$
\left(\hbar \omega_{R, p}\right)^{2}=\frac{2 g_{s} g_{v} \hbar^{2} e^{2}\left|\left\langle u, l ; R\left|v_{x}^{l}\right| g\right\rangle\right|^{2}}{\kappa A R^{2} E_{R}^{u}},
$$

where $g_{v}=2$ is the valley degeneracy. The outer nanotube has dielectric constant

$$
\epsilon_{R^{\prime}}^{l}(\omega)=\kappa+i \kappa^{\prime}
$$

with

$$
\kappa^{\prime}=\frac{2 \pi \sigma_{c}}{R^{\prime} \omega} .
$$

The typical value of $\sigma_{c}$ is $\sim 2\left(e^{2} / h\right)$ for semiconducting outer tubes and $\sim 4\left(e^{2} / h\right)$ for metallic tubes, roughly corresponding to $\kappa^{\prime} \approx 2.5$ and 5 , respectively, for typical double-wall nanotubes.

The effective dynamical conductivity in the vicinity of the exciton energy is calculated as

$$
\begin{aligned}
\operatorname{Re} \tilde{\sigma}_{R}^{\prime}(\omega)= & \frac{\kappa R \omega}{2 \pi\left(1+\alpha^{2}\right) \lambda^{2}} \frac{\left(\hbar \omega_{R, p}\right)^{2}}{2 \hbar \omega_{\text {res }}} \\
& \times \frac{\Gamma}{\left(\hbar \omega-\hbar \omega_{\text {res }}\right)^{2}+\left(\Gamma_{\text {res }} / 2+\Gamma\right)^{2}}, \\
\operatorname{Re} \tilde{\sigma}_{R^{\prime}}^{\prime}(\omega)= & \frac{\tilde{\kappa} R^{\prime} \omega \alpha}{2 \pi\left(1+\alpha^{2}\right)} \\
& \times \frac{\left[\hbar \omega-\hbar \omega_{\text {res }}+\Gamma_{\text {res }} /(2 \alpha)\right]^{2}+\Gamma^{2}}{\left(\hbar \omega-\hbar \omega_{\text {res }}\right)^{2}+\left(\Gamma_{\text {res }} / 2+\Gamma\right)^{2}},
\end{aligned}
$$

giving

$$
\begin{aligned}
\operatorname{Re} \tilde{\sigma}^{\prime}(\omega) & \\
= & \frac{\tilde{\kappa} R^{\prime 2} \omega \alpha}{2 \pi \bar{R}\left(1+\alpha^{2}\right)} \\
& \times \frac{\left[\hbar \omega-\hbar \omega_{\text {res }}+\Gamma_{\text {res }} /(2 \alpha)\right]^{2}+\Gamma^{2}+\left(1+1 / \alpha^{2}\right) \Gamma \Gamma_{\text {res }} / 2}{\left(\hbar \omega-\hbar \omega_{\text {res }}\right)^{2}+\left(\Gamma_{\text {res }} / 2+\Gamma\right)^{2}},
\end{aligned}
$$

where

$$
\begin{gathered}
\left(\hbar \omega_{\text {res }}\right)^{2}=E_{R}^{u 2}+\frac{\kappa}{\tilde{\kappa}}\left[1-\frac{\alpha^{2} \tau}{\left(1+\alpha^{2}\right) \lambda^{2}}\right]\left(\hbar \omega_{R, p}\right)^{2}, \\
\Gamma_{\text {res }}=\frac{\kappa}{\tilde{\kappa}} \frac{\alpha \tau}{\left(1+\alpha^{2}\right) \lambda^{2}} \frac{\left(\hbar \omega_{R, p}\right)^{2}}{\hbar \omega_{\text {res }}}, \\
\lambda=\kappa-\tau(\kappa-1), \\
\alpha=\frac{\kappa^{\prime}}{\tilde{\kappa}} .
\end{gathered}
$$

First, let us consider the case of an ideal tube with $\Gamma=0$. In this case, the absorption of the inner nanotube completely disappears and the spectrum of the outer tube exhibits a clear Fano line shape with asymmetry parameter given by $\alpha$. In fact, the absorption vanishes at

$$
E_{0}=\hbar \omega_{\text {res }}-\frac{\Gamma_{\text {res }}}{2 \alpha},
$$

which always lies lower than $\hbar \omega_{\text {res }}$ because $\alpha>0$ independent of $R^{\prime} / R$. With the increase of $\alpha$ the spectrum becomes closer to a diplike structure.

As discussed above, $\tilde{\kappa}$ gradually decreases from $2 \kappa-1$ at $R^{\prime} / R=1$ to $\kappa$ with increasing $R^{\prime} / R$, but the change remains small for $\kappa \approx 2.5$. Thus, $\alpha$ is nearly independent of $R^{\prime} / R$ and $\alpha \lesssim 1$ for $\kappa^{\prime}=2.5$ and $\alpha \lesssim 2$ for $\kappa^{\prime}=5$. This means that the asymmetry of the line shape remains nearly independent 

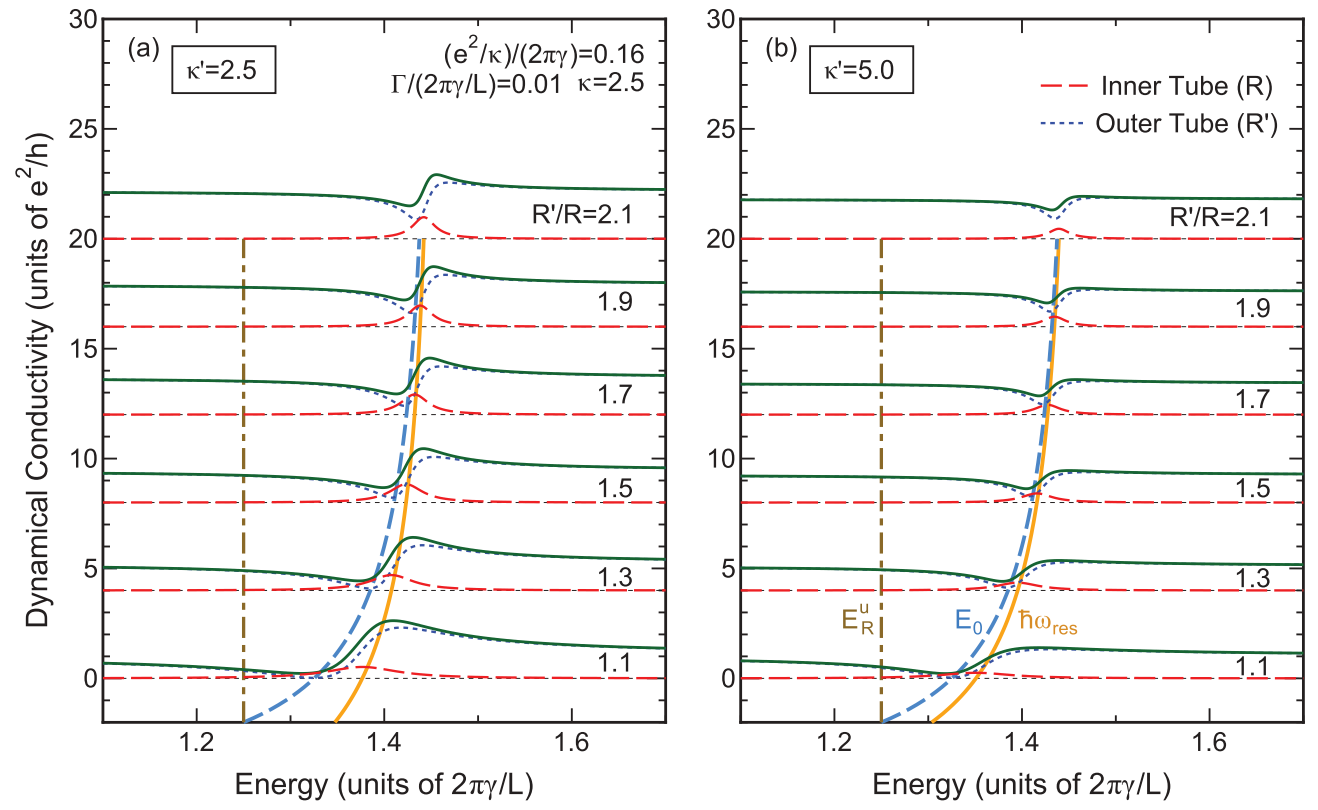

FIG. 5. (Color online) Absorption spectra for (a) $\kappa^{\prime}=2.5$ and (b) $\kappa^{\prime}=5.0$, corresponding to a semiconducting and metallic outer nanotube, together with the characteristic energies, $\hbar \omega_{\text {res }}, E_{0}$, and $E_{R}^{u}$. The energy broadening $\Gamma(2 \pi \gamma / L)^{-1}=0.01$ is used. The solid lines denote $\tilde{\sigma}^{\prime}(\omega)$, dashed $\tilde{\sigma}_{R}^{\prime}(\omega)$, and dotted $\tilde{\sigma}_{R^{\prime}}^{\prime}(\omega)$.

of $R^{\prime} / R$, although it becomes stronger in such a way that the line shape exhibits a diplike structure for $\kappa^{\prime}=5$. Further, broadening $\Gamma_{\text {res }}$ turns out to be nearly the same between $\kappa^{\prime}=$ 2.5 and 5 , but decreases rapidly with increasing $R^{\prime} / R$ because $\tau=\left(R / R^{\prime}\right)^{2}$.

Further, for $R^{\prime} / R=1$, we have $E_{0}=E_{R}^{u}$ and

$$
\left(\hbar \omega_{\mathrm{res}}\right)^{2}=E_{R}^{u 2}+\frac{\kappa}{\tilde{\kappa}\left(1+\alpha^{2}\right)}\left(\hbar \omega_{R, p}\right)^{2} .
$$

With the increase of $R^{\prime} / R$, these energies increase gradually and approach the depolarization-shifted exciton energy in a single tube, i.e., $\hbar \omega_{\text {res }}=E_{0}=\hbar \omega_{R}$, with $\left(\hbar \omega_{R}\right)^{2}=E_{R}^{u 2}+$ $\left(\hbar \omega_{R, p}\right)^{2}$.

This Fano line shape is modified by nonzero level broadening $\Gamma$. The conductivity of the inner tube near the exciton peak becomes nonzero, contributing to the absorption. The peak is symmetric, but exhibits extra broadening $\Gamma_{\text {res }} / 2$ and its integrated intensity is reduced by $\left(1+\alpha^{2}\right)^{-1} \Gamma /\left(\Gamma+\Gamma_{\text {res }} / 2\right)$. The total absorption becomes asymmetric dominated by the Fano line shape of the outer nanotube.

Figure 5 shows examples of absorption spectra of Eqs. (49)(51) for nonzero $\Gamma$, together with the characteristic energies, $\hbar \omega_{\text {res }}, E_{0}$, and $E_{R}^{u}$ for varying $R^{\prime} / R$. In Fig. 5(a), we have $\kappa^{\prime}=2.5$ corresponding to a semiconducting outer tube and in Fig. $5(\mathrm{~b}), \kappa^{\prime}=5$ corresponding to a metallic outer tube. The value of $\Gamma_{\text {res }}$ can be seen from the difference of $\hbar \omega_{\text {res }}$ and $E_{0}$ in Fig. 5 using Eq. (56). We have assumed $\Gamma(2 \pi \gamma / L)^{-1}=$ 0.01 , for which $\Gamma_{\text {res }} / 2 \gg \Gamma$ for $R^{\prime} / R \approx 1$, but $\Gamma_{\text {res }} / 2 \ll \Gamma$ for $R^{\prime} / R \approx 2$.

For $R^{\prime} / R=1.1$ for which $\Gamma_{\text {res }} / 2 \gg \Gamma$, the absorption exhibits almost a pure Fano line shape, becoming vanishingly small at $\hbar \omega=E_{0}$. For $\kappa^{\prime}=5$ in Fig. 5(b), in particular, it is already closer to a diplike structure. With the increase of $R^{\prime} / R, \Gamma_{\text {res }}$ becomes smaller and the line shape deviates from the Fano line shape because of dominant $\Gamma$. In fact, the minimum conductivity becomes nonzero and the structure itself becomes smaller in comparison with frequency-independent background absorption.

The Fano line shape obtained in the model qualitatively explains the features of the results shown in Figs. 2 and 4. In fact, the degree of the asymmetry for $R^{\prime} / R=1.5$ shown in Fig. 5(a) is similar to that of the asymmetric peak around $\hbar \omega(2 \pi \gamma / L)^{-1} \sim 1.38$ for a semiconducting outer tube shown in Fig. 2. The near-diplike structure for $R^{\prime} / R=1.5$ shown in Fig. 5(b) is similar to that around $\hbar \omega(2 \pi \gamma / L)^{-1} \sim 1.34$ for a metallic outer tube shown in Fig. 4. Quantitatively, however, the model is insufficient because the depolarization-shifted peak lies very close to the bottom of the interband continuum.

Because of the asymmetric Fano line shape, it is difficult to determine the exciton energy of the inner nanotube from the total absorption. Therefore, we shall use the peak of the conductivity of the inner nanotube to identify the exciton energy in the following.

\section{DIAMETER DEPENDENCE}

In double-wall tubes, the interwall distance is considered to be about the same as that of interlayer distance in bulk graphite. Thus, in order to discuss the diameter dependence for comparison with experiments, we calculate excitation energies and optical absorption spectra for varying diameter with the interwall distance fixed to $\left|R^{\prime}-R\right|=0.34 \mathrm{~nm}$.

Figure 6 shows calculated absorption spectra including depolarization effect, $\operatorname{Re} \tilde{\sigma}^{\prime}(\omega)$, for various combinations of two nanotubes. The double-wall tube consists of two semiconducting tubes $\left(R<R^{\prime}\right)$ in (a), a semiconducting inner tube and a metallic outer tube $\left(R<R^{\prime}\right)$ in (b), and a 


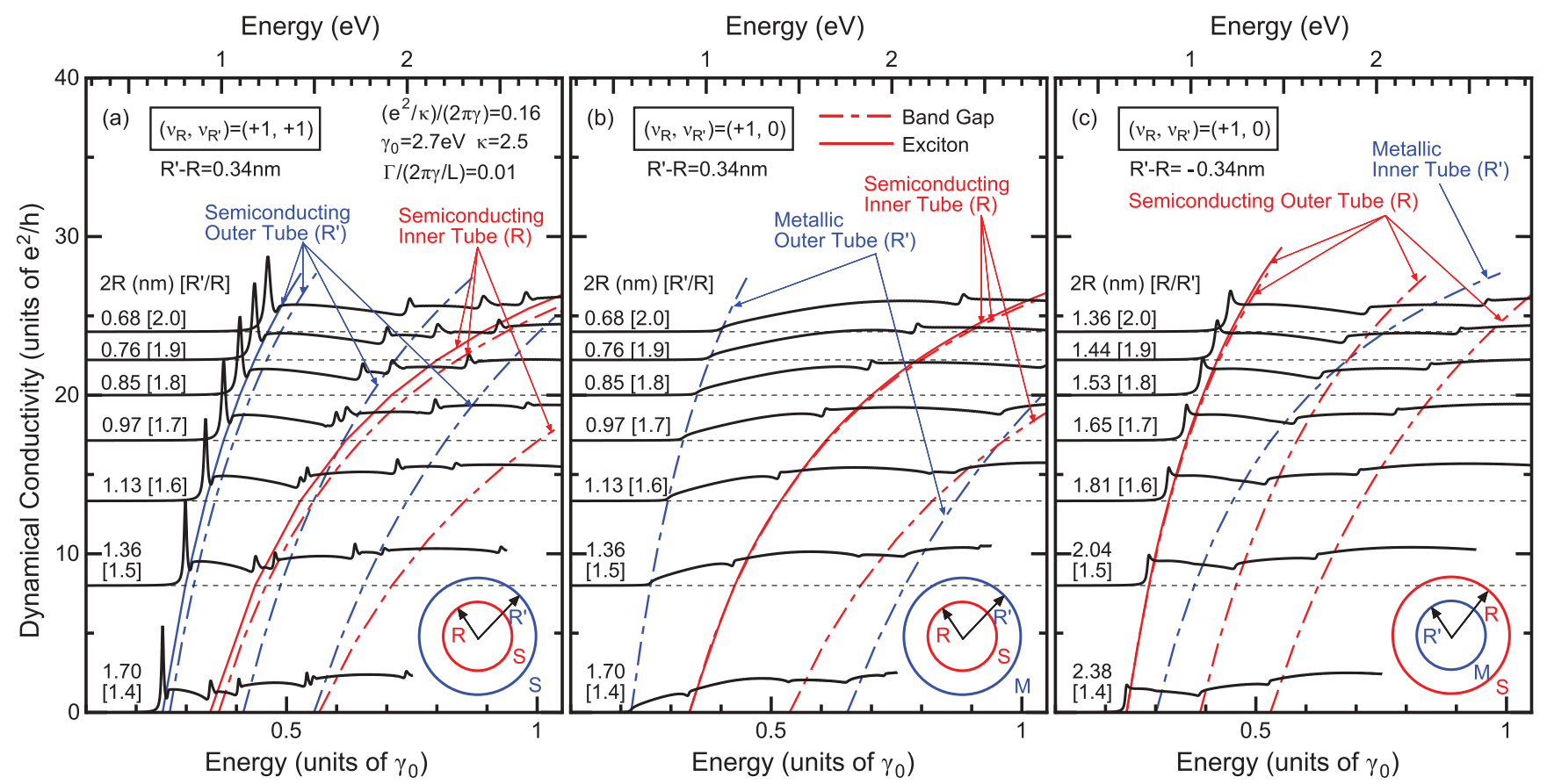

FIG. 6. (Color online) Calculated effective dynamical conductivity $\tilde{\sigma}^{\prime}(\omega)$ of a double-wall nanotube with the fixed interwall distance at $R^{\prime}-$ $R= \pm 0.34 \mathrm{~nm}$, consisting of (a) two semiconducting tubes, (b) a semiconducting inner tube and a metallic outer tube, and (c) a metallic inner tube and a semiconducting outer tube. The tube diameter is varied from $2 R=0.68 \mathrm{~nm}$ to $1.7 \mathrm{~nm}$ in (a) and (b) and $2 R=1.36 \mathrm{~nm}$ to $2.38 \mathrm{~nm}$ in (c). The solid and dash-dotted lines represent the position of exciton peak with depolarization and the bottoms of the interband continua, respectively.

metallic inner tube and a semiconducting outer tube $\left(R^{\prime}<R\right)$ in (c).

In Fig. 6(a) for two semiconducting tubes, the result for diameter $2 R=1.70 \mathrm{~nm}$ is the same as in Fig. 2. With the decrease in the diameter, $R^{\prime} / R$ increases from 1.4 to 2 for $2 R=0.68 \mathrm{~nm}$ and, as the ratio of interwall distance to tube radius increases, effects of interwall screening are reduced accordingly. In fact, the Fano line shape associated with the exciton of the inner tube becomes less asymmetric, although the change is only gradual. We can see a complex line shape for $2 R=1.13 \mathrm{~nm}$ around $\hbar \omega / \gamma_{0}=0.55$, due to Fano interference between the exciton of the first band gap in the inner tube and that of the second band gap in the outer tube lying close to each other.

In Fig. 6(b) for inner semiconducting and outer metallic tubes, the result for diameter $2 R=1.70 \mathrm{~nm}$ is the same as in Fig. 4. In this case, the reduction of interwall screening appears more clearly in the change in the Fano line shape of the exciton of the inner tube. In fact, the diplike structure for $2 R=1.70 \mathrm{~nm}$ changes into the structure of a more symmetric peak for $2 R=0.68 \mathrm{~nm}$.

When an outer semiconducting tube contains an inner metallic tube as shown in Fig. 6(c), the exciton peak of the semiconducting tube becomes much weaker than in Fig. 6(a). Note that the diameter of the outer semiconducting tube, $2 R^{\prime}$, in (a) corresponds to $2 R$ in (c). This is mainly due to large reduction in the exciton binding energy caused by metallic screening of the inner tube as in the case of parallel polarization. ${ }^{27}$ The decrease of the diameter leads to slight enhancement of the exciton peak due to the reduction of interwall screening. In contrast, the inner metallic nanotube causes essentially no structure in the total absorption spectrum.
Figure 7 shows the exciton energies and the bottoms of the interband continua as a function of the tube diameter, corresponding to Fig. 6. The energy of exciton with depolarization is determined from the peak position of the absorption in each nanotube. The lower horizontal axis shows diameter $2 R$ of the semiconducting tube and the upper horizontal axis shows diameter $2 R^{\prime}$ of the outer semiconducting tube in (a), $R^{\prime} / R$ in (b), and diameter $2 R^{\prime}$ of inner metallic tube in (c).

In Fig. 7(a) for two semiconducting tubes, the shift due to interwall screening is considerable for the band gap, while that is almost negligible in this scale for the exciton without depolarization. With depolarization effect taken into account, the exciton remains well separated from the interband continuum, although exhibiting appreciable amount of red shift. In Figs. 7(b) and 7(c), there is no exciton in the metallic tube and the exciton of the semiconducting tube with depolarization almost merges into the bottom of the interband continuum due to interwall screening.

Figure 8 shows the shift of excitation energies due to the presence of an outer or inner tube. The diameter dependence is qualitatively the same for any combinations of tubes. The exciton without depolarization shows almost a constant red shift independent of the diameter, while the band gap exhibits comparatively larger shift, which decreases with the increase of the diameter. These behaviors are quite similar, even quantitatively, to those for parallel polarization. ${ }^{27}$ The depolarization effect clearly enhances the red shift of the exciton due to the interwall screening up to about a half of that of the band gap.

For a double-wall tube consisting of two semiconducting tubes in (a), the shift of the exciton energy of the inner tube lies between 40 and $70 \mathrm{meV}$ and the outer tube between 30 


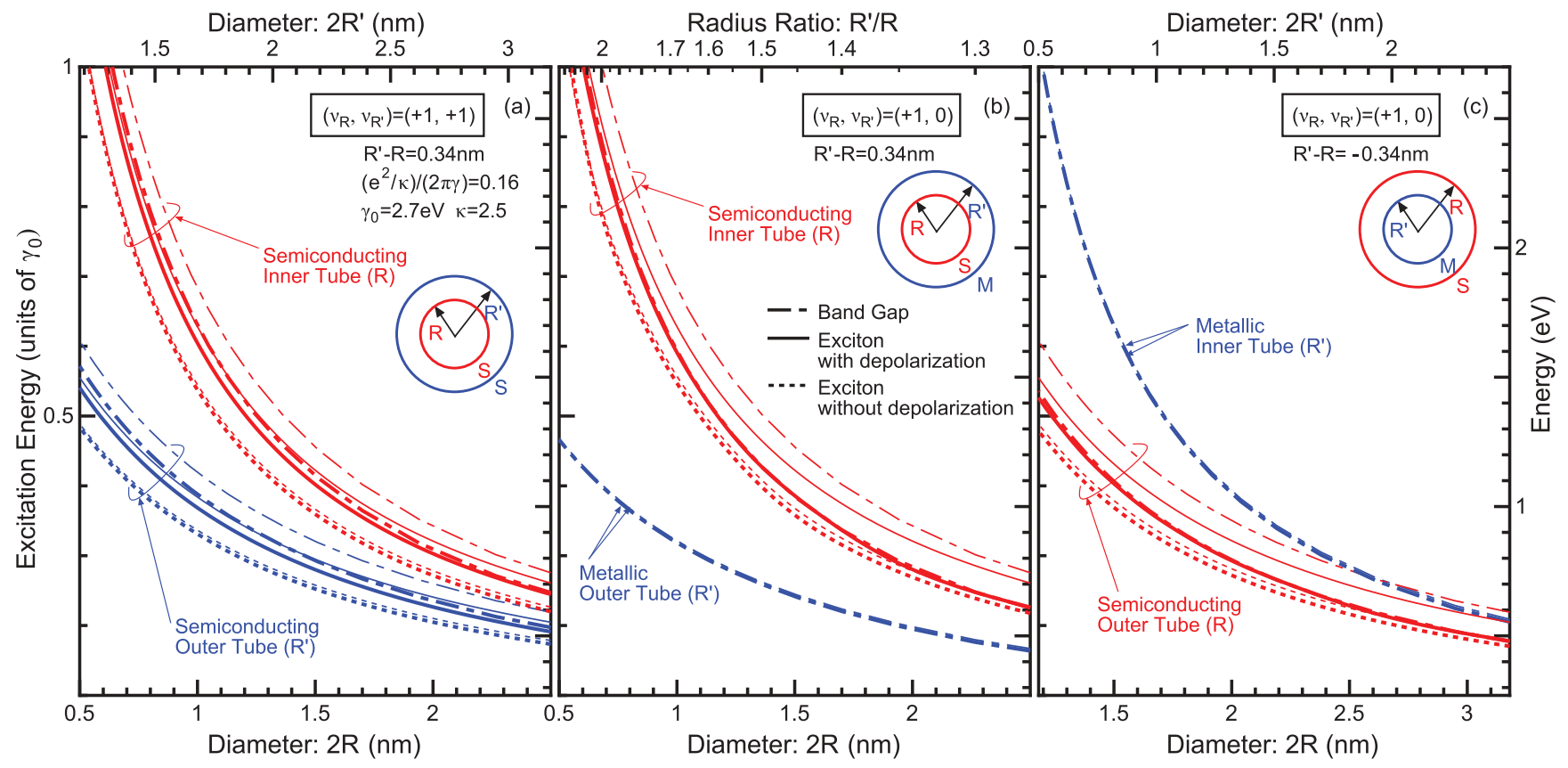

FIG. 7. (Color online) The exciton energies and the bottoms of the interband continua for the fixed interwall distance at $R^{\prime}-R= \pm 0.34 \mathrm{~nm}$ as a function of the tube diameter, (a) consisting of both semiconducting tubes, (b) a semiconducting tube surrounded by a metallic outer tube, and (c) a semiconducting tube surrounding a metallic inner tube. The thick (thin) solid, dotted, and dash-dotted lines represent the exciton energies with depolarization, those without depolarization, and the bottoms of the interband continua in double-wall (single-wall) tubes, respectively.

and $40 \mathrm{meV}$. When the semiconducting tube is surrounded by a metallic outer tube in (b), the shift lies between 80 and $95 \mathrm{meV}$. When it contains a metallic inner tube in (c), the shift lies between 65 and $80 \mathrm{meV}$.

\section{DISCUSSION AND SUMMARY}

We point out that the Fano effect in absorption spectra can appear even in single-wall nanotubes. For example, it results from the coupling between an exciton state of the second gap and a continuum state of the first gap. To see this by a simple model, suppose that the conductivity in the single-wall tube with radius $R$ is given by

$$
\sigma_{R}^{l}(\omega)=\frac{\kappa R \omega}{2 \pi i} \frac{\left(\hbar \omega_{R, p}\right)^{2}}{E_{R}^{u 2}-(\hbar \omega)^{2}-2 i \hbar \omega \Gamma}+\sigma_{c},
$$

around the exciton energy $E_{R}^{u}$ where the first term represents the exciton resonance and the second constant $\sigma_{c}$ denotes a contribution from the continuum state. After the depolarization effect is taken into account, the effective dynamical conductivity defined by $\tilde{\sigma}_{R}^{\prime}(\omega)=\left(\kappa^{2} / 2\right) \sum_{l= \pm 1} \sigma_{R}^{l}(\omega) /\left|\epsilon_{R}^{l}(\omega)\right|^{2}$ can

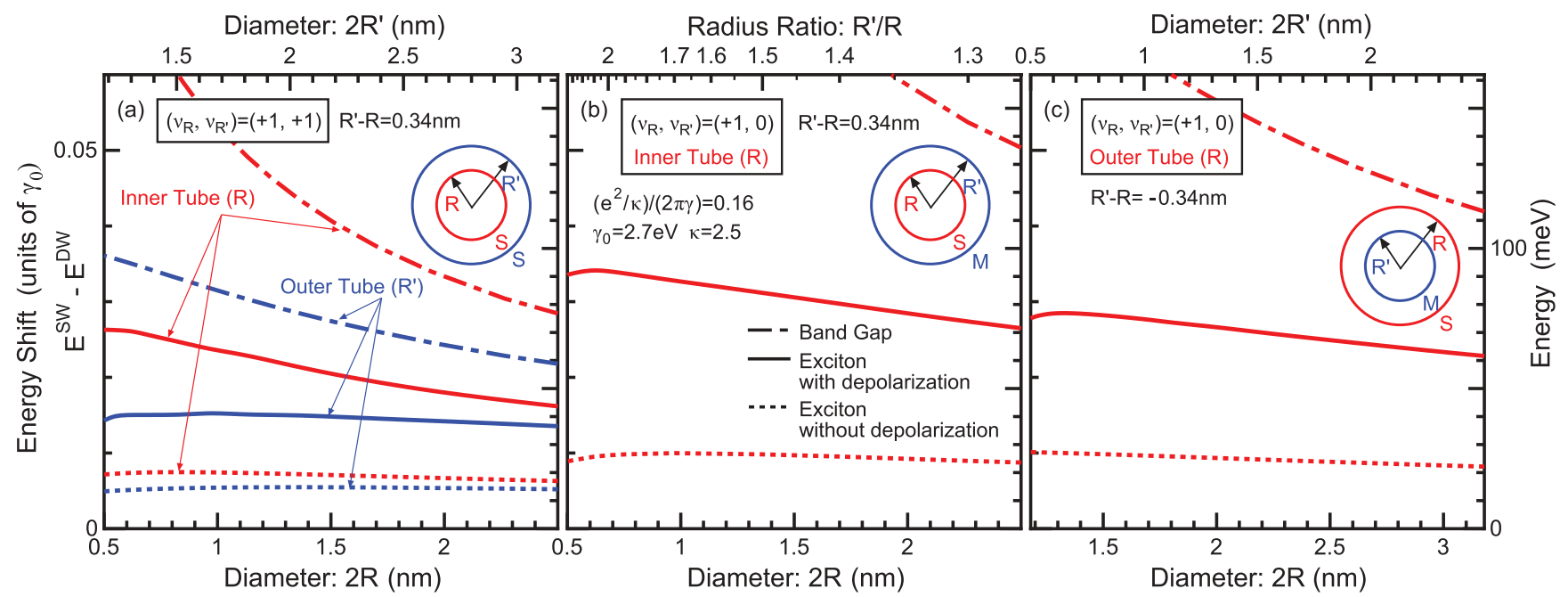

FIG. 8. (Color online) The shift of excitation energies due to the presence of an outer or inner tube for the fixed interwall distance at $R^{\prime}-R= \pm 0.34 \mathrm{~nm}$ as a function of the tube diameter, (a) consisting of both semiconducting tubes, (b) a semiconducting tube surrounded by a metallic outer tube, and (c) a semiconducting tube surrounding a metallic inner tube. The solid, dotted, and dash-dotted lines represent those of the exciton energies with depolarization, those without depolarization, and the bottoms of the interband continua, respectively. 
be written in the same form as Eq. (51) by putting $R=R^{\prime}$ and $2 \kappa-1 \rightarrow \kappa$. In fact, in Fig. 4 of Ref. 31, calculated absorption spectra of semiconducting single-wall nanotubes clearly show the Fano behavior of the exciton peak of the second gap $\left[\hbar \omega(2 \pi \gamma / L)^{-1} \simeq 2.2\right.$ for $\left(e^{2} / \kappa L\right) /(2 \pi \gamma / L)=$ 0.1 and $\hbar \omega(2 \pi \gamma / L)^{-1} \simeq 2.5$ for $\left.\left(e^{2} / \kappa L\right) /(2 \pi \gamma / L)=0.25\right]$.

In summary, we have studied effects of interwall screening on optical absorption spectra for cross-polarization geometry in double-wall carbon nanotubes in the static screened HartreeFock approximation within a $\boldsymbol{k} \cdot \boldsymbol{p}$ scheme. Interwall screening effects lead to the reduction of exciton binding energies and band gaps and, as a consequence, the exciton shows a significant red shift which is larger than that in the parallelpolarization geometry. The red shift slightly decreases as the diameter increases and is estimated to be of the order of $50 \mathrm{meV}$.

In a double-wall nanotube composed of two semiconducting tubes, the depolarization effect for the exciton in the outer tube is qualitatively the same as in the single-wall tube except that the interwall screening causes the red shift and the slight intensity reduction, while that for the inner tube gives rise to the spectral interference between the outer and the inner tubes and the exciton peak of the inner tube is modified into an asymmetric Fano line shape.

In a semiconducting tube surrounded by or containing a metallic tube, the exciton binding energy is strongly reduced by the metallic interwall screening. Only a weak Fano-like feature appears at the exciton peak for the inner semiconducting tube and a feature such as interband continuum appears for the outer semiconducting tube. There is no prominent peak structure in the total absorption associated with the exciton in a metallic nanotube.

\section{ACKNOWLEDGMENTS}

This work was supported in part by a Grant-in-Aid for Scientific Research on Innovative Area "Optical Science of Dynamically Correlated Electrons" (Grant No. 20104009), by a Grant-in-Aid for Scientific Research on Priority Area "Carbon Nanotube Nanoelectronics" (Grant No. 19054004), and by Grants-in-Aid for Scientific Research (C) (Grants No. 20540307 and No. 22540326) from the Ministry of Education, Culture, Sports, Science and Technology, Japan.

\section{APPENDIX A: ELECTRIC FIELD IN MULTIWALL NANOTUBES}

In a nanotube with circumference $L_{i}=2 \pi R_{i}$, induced current $j_{i x}^{l}$ in the circumference direction and induced charge density $\rho_{i}^{l}$ satisfy continuity equation ${ }^{31}$

$$
\rho_{i}^{l}=\frac{2 \pi l}{L_{i} \omega} j_{i x}^{l}
$$

which contributes to the electrostatic potential in wall $i$

$$
\phi_{i}^{l}(\theta)=\frac{1}{e^{2}} \sum_{j} L_{j} V_{i j}^{0}(l, 0) \rho_{j}^{l} e^{i l \theta},
$$

with

$$
V_{i j}^{0}(l, 0)=\frac{e^{2}}{|l|}\left(\frac{R_{\min }}{R_{\max }}\right)^{|l|} .
$$

This gives polarization

$$
P_{i x}^{l}=\frac{1}{2 L_{i}} e^{-i l \theta} \frac{\partial}{\partial \theta} \phi_{i}^{l}(\theta)=\frac{i l}{2 e^{2}} \sum_{j} \frac{R_{j}}{R_{i}} V_{i j}^{0}(l, 0) \rho_{j}^{l} .
$$

The external field and the induced polarization determine the electric field in the circumference direction through $E_{i x}^{l}=$ $D_{i x}^{l}-4 \pi P_{i x}^{l}$. Using $j_{i x}^{l}=\sigma_{i}^{l}(\omega) E_{i x}^{l}$, we get

$$
E_{i x}^{l}=D_{i x}^{l}-\sum_{j} \frac{R_{j}}{R_{i}} \beta_{i j}(l, 0)\left[\epsilon_{j}^{l}(\omega)-1\right] E_{j x}^{l},
$$

where $\beta_{i j}(l, 0)=V_{i j}^{0}(l, 0) / V_{j j}^{0}(l, 0)=\left(R_{\min } / R_{\max }\right)^{|l|}$ and $\epsilon_{j}^{l}(\omega)$ is given by Eq. (24). Using the dielectric matrix of Eq. (11), we can rewrite Eq. (A5) as Eq. (22).

\section{APPENDIX B: RELATION TO ENVIRONMENT EFFECTS}

When the outer tube with radius $R^{\prime}$ is regarded as a dielectric material with dielectric constant $\kappa_{\text {out }}$ [i.e., $\epsilon_{R^{\prime}}^{l}(\omega)=\kappa_{\text {out }}$ ], $\tilde{\epsilon}_{R}^{l}(\omega)$ of Eq. (39) becomes

$$
\begin{gathered}
\tilde{\epsilon}_{R}^{l}(\omega)=\kappa_{\text {out }}\left[\kappa\left(1-p_{l}\right)+\frac{2 \pi i|l| q_{l}}{R \omega} \sigma_{R}^{l}(\omega)\right], \\
p_{l}=\left(1-\frac{1}{\kappa}\right)\left(1-q_{l}\right), \\
q_{l}=1-\frac{\delta_{\kappa}}{1+\delta_{\kappa}}\left(\frac{R}{R^{\prime}}\right)^{2|l|}, \\
\delta_{\kappa}=\kappa_{\text {out }}-1 .
\end{gathered}
$$

We obtain the same form as in Ref. 65 for outside dielectric material except that $\delta_{\kappa}$ in Eq. (B3) is replaced with $\delta_{\kappa} / 2$ in Ref. 65, when we identify the electric field at the inner tube surface, $D_{x}^{l} / \kappa_{\text {out }}$, as the external field.

On the other hand, when the inner tube with radius $R$ is a dielectric material with dielectric constant $\kappa_{\text {in }}$ [i.e., $\epsilon_{R}^{l}(\omega)=$ $\left.\kappa_{\text {in }}\right], \tilde{\epsilon}_{R^{\prime}}^{l}(\omega)$ of Eq. (40) becomes

$$
\begin{gathered}
\tilde{\epsilon}_{R^{\prime}}^{l}(\omega)=\frac{1}{q_{l}^{\prime}}\left[\kappa\left(1-p_{l}^{\prime}\right)+\frac{2 \pi i|l| q_{l}^{\prime}}{R^{\prime} \omega} \sigma_{R^{\prime}}^{l}(\omega)\right], \\
p_{l}^{\prime}=\left(1-\frac{1}{\kappa}\right)\left(1-q_{l}^{\prime}\right), \\
q_{l}^{\prime}=1-\frac{\delta_{\kappa}^{\prime}}{1+\delta_{\kappa}^{\prime}}\left(\frac{R}{R^{\prime}}\right)^{2|l|}, \\
\delta_{\kappa}^{\prime}=\kappa_{\mathrm{in}}-1 .
\end{gathered}
$$

We obtain the same form as in Ref. 65 for inside material except that $\delta_{\kappa}^{\prime}$ in Eq. (B7) is again replaced with $\delta_{\kappa}^{\prime} / 2$.

This factor 1/2 appearing in Ref. 65 is the depolarization factor for cylindrical dielectric object. It does not appear in the present system because the nanotube responds only to the electric field component parallel to the cylinder surface. 
*tomio@eng.hokudai.ac.jp

${ }^{1}$ R. Saito, G. Dresselhaus, and M. S. Dresselhaus, Physical Properties of Carbon Nanotubes (Imperial College Press, London, 1998). ${ }^{2}$ T. Ando, J. Phys. Soc. Jpn. 74, 777 (2005).

${ }^{3}$ A. H. Castro Neto, F. Guinea, N. M. Peres, K. S. Novoselov, and A. K. Geim, Rev. Mod. Phys. 81, 109 (2009).

${ }^{4}$ T. Ando, J. Phys. Soc. Jpn. 66, 1066 (1997).

${ }^{5}$ T. Ando, J. Phys. Soc. Jpn. 73, 3351 (2004).

${ }^{6}$ V. Perebeinos, J. Tersoff, and Ph. Avouris, Phys. Rev. Lett. 92, 257402 (2004).

${ }^{7}$ M. Ichida, S. Mizuno, Y. Tani, Y. Saito, and A. Nakamura, J. Phys. Soc. Jpn. 68, 3131 (1999).

${ }^{8}$ F. Wang, G. Dukovic, L. E. Brus, and T. F. Heinz, Science 308, 838 (2005).

${ }^{9}$ J. Maultzsch, R. Pomraenke, S. Reich, E. Chang, D. Prezzi, A. Ruini, E. Molinari, M. S. Strano, C. Thomsen, and C. Lienau, Phys. Rev. B 72, 241402 (2005).

${ }^{10}$ Y. Kanemitsu, Phys. Chem. Chem. Phys. 13, 14879 (2011).

${ }^{11}$ C. D. Spataru, S. Ismail-Beigi, L. X. Benedict, and S. G. Louie, Phys. Rev. Lett. 92, 077402 (2004).

${ }^{12}$ J. Deslippe, M. Dipoppa, D. Prendergast, M. V. O. Moutinho, R. B. Capaz, and S. G. Louie, Nano. Lett. 9, 1330 (2009).

${ }^{13}$ H. Hirori, K. Matsuda, and Y. Kanemitsu, Phys. Rev. B 78, 113409 (2008).

${ }^{14}$ D. Shimamoto, H. Muramatsu, T. Hayashi, Y. A. Kim, M. Endo, J. S. Park, R. Saito, M. Terrones, and M. S. Dresselhaus, Appl. Phys. Lett. 94, 083106 (2009).

${ }^{15}$ Y. Ohno, S. Iwasaki, Y. Murakami, S. Kishimoto, S. Maruyama, and T. Mizutani, Phys. Rev. B 73, 235427 (2006).

${ }^{16}$ Y. Ohno, S. Iwasaki, Y. Murakami, S. Kishimoto, S. Maruyama, and T. Mizutani, Phys. Stat. Sol. B 244, 4002 (2007).

${ }^{17}$ Y. Miyauchi, R. Saito, K. Sato, Y. Ohno, S. Iwasaki, T. Mizutani, J. Jiang, and S. Maruyama, Chem. Phys. Lett. 442, 394 (2007).

${ }^{18}$ T. Ando, J. Phys. Soc. Jpn. 79, 024706 (2010).

${ }^{19}$ C. Shen, A. H. Brozena, and Y. H. Wang, Nanoscale 3, 503 (2011).

${ }^{20}$ M. Endo, H. Muramatsu, T. Hayashi, Y. A. Kim, M. Terrones, and M. S. Dresselhaus, Nature (London) 433, 476 (2005).

${ }^{21}$ T. Okazaki, S. Bandow, G. Tamura, Y. Fujita, K. Iakoubovskii, S. Kazaoui, N. Minami, T. Saito, K. Suenaga, and S. Iijima, Phys. Rev. B 74, 153404 (2006).

${ }^{22}$ H. Muramatsu, T. Hayashi, Y. A. Kim, D. Shimamoto, M. Endo, V. Meunier, B. G. Sumpter, M. Terrones, and M. S. Dresselhaus, Small 5, 2678 (2009).

${ }^{23}$ A. A. Green and M. C. Hersam, Nature Nano. 4, 64 (2009).

${ }^{24}$ D. A. Tsyboulski, Y. Hou, N. Fakhri, S. Ghosh, R. Zhang, S. M. Bachilo, M. Pasquali, L. Chen, J. Liu, and R. B. Weisman, Nano. Lett. 9, 3282 (2009).

${ }^{25}$ F. Villalpando-Paez, L. G. Moura, C. Fantini, H. Muramatsu, T. Hayashi, Y. A. Kim, M. Endo, M. Terrones, M. A. Pimenta, and M. S. Dresselhaus, Phys. Rev. B 82, 155416 (2010).

${ }^{26}$ M. V. Shuba, G. Ya. Slepyan, S. A. Maksimenko, C. Thomsen, and A. Lakhtakia, Phys. Rev. B 79, 155403 (2009).

${ }^{27}$ Y. Tomio, H. Suzuura, and T. Ando, Phys. Rev. B 85, 085411 (2012).

${ }^{28}$ E. Chang, G. Bussi, A. Ruini, and E. Molinari, Phys. Rev. Lett. 92, 196401 (2004).

${ }^{29}$ H. Zhao and S. Mazumdar, Phys. Rev. Lett. 93, 157402 (2004).

${ }^{30}$ J. Lefebvre and P. Finnie, Phys. Rev. Lett. 98, 167406 (2007).
${ }^{31}$ S. Uryu and T. Ando, Phys. Rev. B 74, 155411 (2006).

${ }^{32}$ S. Uryu and T. Ando, AIP Conf. Proc. 893, 1033 (2007).

${ }^{33}$ S. Kilina, S. Tretiak, S. K. Doorn, Z. Luo, F. Papadimitrakopoulos, A. Piryatinski, A. Saxena, and A. R. Bishop, Proc. Natl. Acad. Sci. USA 105, 6797 (2008).

${ }^{34}$ Y. Miyauchi, H. Ajiki, and S. Maruyama, Phys. Rev. B 81, 121415 (2010).

${ }^{35}$ S. Uryu and T. Ando, Phys. Rev. B 83, 085404 (2011).

${ }^{36}$ Y. Miyauchi, M. Oba, and S. Maruyama, Phys. Rev. B 74, 205440 (2006).

${ }^{37}$ J. W. McClure, Phys. Rev. 104, 666 (1956).

${ }^{38}$ J. C. Slonczewski and P. R. Weiss, Phys. Rev. 109, 272 (1958).

${ }^{39}$ D. P. DiVincenzo and E. J. Mele, Phys. Rev. B 29, 1685 (1984).

${ }^{40}$ G. W. Semenoff, Phys. Rev. Lett. 53, 2449 (1984).

${ }^{41}$ H. Ajiki and T. Ando, J. Phys. Soc. Jpn. 62, 1255 (1993).

${ }^{42}$ C. L. Kane and E. J. Mele, Phys. Rev. Lett. 78, 1932 (1997).

${ }^{43}$ M. Kociak, K. Suenaga, K. Hirahara, Y. Saito, T. Nakahira, and S. Iijima, Phys. Rev. Lett. 89, 155501 (2002).

${ }^{44}$ J. M. Zuo, I. Vartanyants, M. Gao, R. Zhang, and L. A. Nagahara, Science 300, 1419 (2003).

${ }^{45}$ Y.-G. Yoon, P. Delaney, and S. G. Louie, Phys. Rev. B 66, 073407 (2002).

${ }^{46}$ F. Triozon, S. Roche, A. Rubio, and D. Mayou, Phys. Rev. B 69, 121410(R) (2004).

${ }^{47}$ S. Uryu and T. Ando, Phys. Rev. B 72, 245403 (2005).

${ }^{48}$ J.-C. Charlier, X. Blase, and S. Roche, Rev. Mod. Phys. 79, 677 (2007).

${ }^{49}$ H. Suzuura and T. Ando, Phys. Rev. Lett. 89, 266603 (2002).

${ }^{50}$ T. Ando, J. Phys. Soc. Jpn. 75, 054701 (2006).

${ }^{51}$ L. Wang, P. S. Davids, A. Saxena, and A. R. Bishop, Phys. Rev. B 46, 7175 (1992).

${ }^{52}$ M. F. Lin and Kenneth W.-K. Shung, Phys. Rev. B 47, 6617 (1993).

${ }^{53}$ P. J. Lin-Chung and A. K. Rajagopal, Phys. Rev. B 49, 8454 (1994).

${ }^{54}$ R. Perez and W. Que, J. Phys.: Condens. Matter 18, 3197 (2006).

${ }^{55}$ Y. H. Ho, G. W. Ho, S. C. Chen, J. H. Ho, and M. F. Lin, Phys. Rev. B 76, 115422 (2007).

${ }^{56}$ B. Scharf, J. Fabian, and A. Matos-Abiague, Phys. Rev. B 81, 125428 (2010).

${ }^{57}$ C. D. Spataru, S. Ismail-Beigi, R. B. Capaz, and S. G. Louie, Phys. Rev. Lett. 95, 247402 (2005).

${ }^{58}$ T. Ando, J. Phys. Soc. Jpn. 75, 024707 (2006).

${ }^{59}$ Y. Jia, G. Yu, and J. Dong, Eur. Phys. J. B 61, 433 (2008).

${ }^{60}$ T. Oshima and H. Suzuura, AIP Conf. Proc. 1399, 807 (2011).

${ }^{61}$ R. Kubo, J. Phys. Soc. Jpn. 12, 570 (1957).

${ }^{62}$ H. Ajiki and T. Ando, Physica B 201, 349 (1994).

${ }^{63}$ H. Ajiki and T. Ando, Jpn. J. Appl. Phys. 34, 107 (1995).

${ }^{64}$ M. Yamamoto, M. Koshino, and T. Ando, J. Phys. Soc. Jpn. 77, 084705 (2008)

${ }^{65}$ S. Uryu and T. Ando, Phys. Rev. B 86, 125412 (2012).

${ }^{66}$ T. Ando, Physica E 43, 645 (2011).

${ }^{67}$ S. Uryu, H. Ajiki, and T. Ando, Phys. Rev. B 78, 115414 (2008).

${ }^{68}$ S. Uryu and T. Ando, Phys. Rev. B 77, 205407 (2008).

${ }^{69}$ F. Wang, D. J. Cho, B. Kessler, J. Deslippe, P. J. Schuck, S. G. Louie, A. Zettl, T. F. Heinz, and Y. R. Shen, Phys. Rev. Lett. 99, 227401 (2007)

${ }^{70}$ T. Ando and T. Nakanishi, J. Phys. Soc. Jpn. 67, 1704 (1998).

${ }^{71}$ T. Ando, T. Nakanishi, and R. Saito, J. Phys. Soc. Jpn. 67, 2857 (1998).

${ }^{72}$ T. Ando and H. Suzuura, J. Phys. Soc. Jpn. 71, 2753 (2002). 